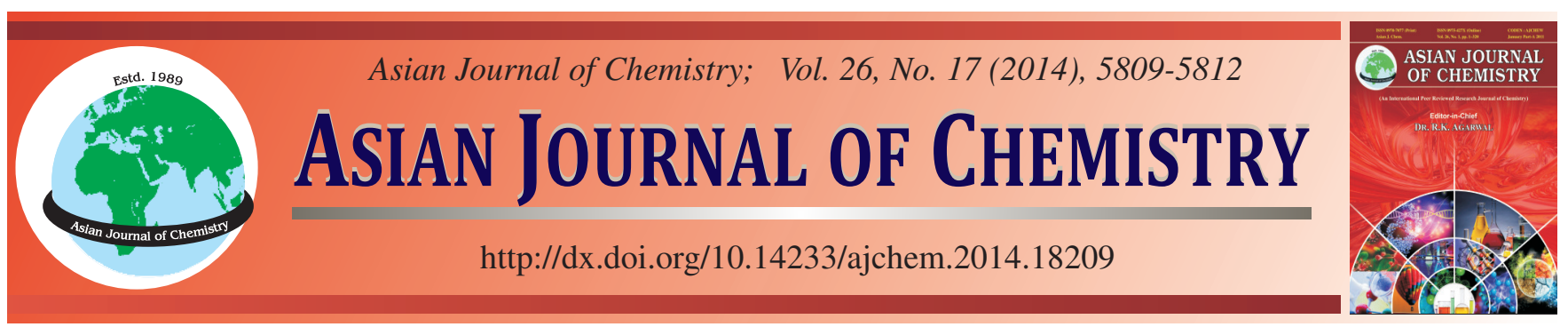

\title{
Preparation and Properties of Amorphous Calcium Phosphate/ Multi(amino Acid) Copolymer Composite for Bone Repair $\dagger$
}

\author{
H.B. SHAO ${ }^{1}$, Y.M. YAO ${ }^{2}$, L. ZHANG ${ }^{3}{ }^{*}$, Q.F. ZHANG ${ }^{1}$ and H. $\mathrm{LI}^{4}$
}

${ }^{1}$ First Hospital of Hebei Medical University, Hebei Province, Shijiazhuang 050031, P.R. China

${ }^{2}$ Cardiovascular Disease Hospital in Shijiazhuang, Hebei Province, Shijiazhuang 050037, P.R. China

${ }^{3}$ Hebei Medical University, Hebei Province, Shijiazhuang 050037, P.R China

${ }^{4}$ College of Physical Science and Technology, Sichuan Province, Chengdu 610041, P.R China

*Corresponding author: E-mail: yyming7209y@sohu.com

In the present study, a novel bioactive composite composed of amorphous calcium phosphate (ACP) and multi (amino acid) copolymer (MAC) was fabricated for bone repair and characterized. The results show that the compressive strength of the ACP/MAC composites was in the range of 72-96 MPa, increased with an increase in the amorphous calcium phosphate content. The composite was degradable and kept a sustain degradation rate with the weight loss of 65-84 w \% after soaking in phosphate buffered saline (PBS) for 24 weeks, significantly increased with an increase in the ACP content. In vitro MG63 cell co-culture experiments showed that the composite shows good cytocompatibility and thus allows cells to adhere, proliferate and differentiate.

Keywords: Amorphous calcium phosphate, Composite, Degradation, Cytocompatibility.

\section{INTRODUCTION}

Amorphous calcium phosphate $\left[\mathrm{ACP}, \mathrm{Ca}_{3}\left(\mathrm{PO}_{4}\right)_{2} \cdot 3 \mathrm{H}_{2} \mathrm{O}\right]$ has being extensively investigated as dental active restorative materials in recent years. For example, it usually compounded with polymeric resins and is used as restorations in small carious lesions and to seal pits and fissures in teeth where plaque can accumulate and lead to caries and as adhesives in orthodontics and help provent the demineralization of tooth enamel $^{1-3}$. Furthermore, ACP has also been widely used as bone repair materials, such as coatings on metal implants and as bone cements due to their outstanding biocompatibility ${ }^{4,5}$. In addition, as an intermediate phase in the preparation of several micelles of calcium phosphate by precipitation, ACP shows relatively high solubility and unstable crystalline structure in aqueous environments, which lead to faster release of calcium and phosphate ions than hydroxyapatite (HA) and tricalcium phosphate (TCP) and hence shows better bioactivity than HA and $\mathrm{TCP}^{4,5}$. It is obvious that ACP is a good candidate for the preparation of degradable Ca-P bioactive material/polymer composites for bone reconstruction and regeneration.

Recently, a series of new degradable multi (amino acid) copolymers (MAC) were developed for biomedical application $^{6-8}$. The degradation of MAC had no significant effects on the $\mathrm{pH}$ of the ambient environment and the material maintained adequate mechanical strength during the degradation period. Both in vitro cell culture and in vivo implantation experiments showed that MAC exhibits good biocompatibility. Additionally, we have developed composites by combination of MAC and calcium deficient hydroxyapatite (CDHA) and MAC and ACP and it was found that the composites have good mechanical properties and is degradable ${ }^{9,10}$. However, our further experiments found that MAC, CDHA/MAC and ACP/ MAC composites showed poor degradability, especially in vivo degradation.

In order to obtain new composite for bone repair with improved degradation properties, in the present study, we prepared a novel composite composed of ACP and MAC (ACP/ $\mathrm{MAC})$. In addition, the mechanical properties, degradability and biocompatibility of ACP/MAC were evaluated with the $\mathrm{HA} / \mathrm{MAC}$ as the control.

\section{EXPERIMENTAL}

The preparation method of ACP was described in the previous study ${ }^{11}$. The ACP/MAC composite was synthesized through an in situ polymerization method as reported by $\mathrm{Li}$ et al. ${ }^{10}$. ACP/MAC composites with ACP contents of 10, 20, 
30 and $40 \mathrm{w} \%$ were prepared and denoted 10ACP/MAC, 20ACP/MAC, 30ACP/MAC and 40ACP/MAC, respectively.

Detection method: The composition of the 30TCP/MAC composite was characterized by fourier transform infrared spectroscopy (170SX FT-IR Spectrometer, Nicolet) and the compressive strength of the composites (with a size of $10 \mathrm{~mm}$ $\times 10 \mathrm{~mm} \times 20 \mathrm{~mm}$ ) were analyzed using a mechanical testing machine (REGER 30-50, Shenzhen Reger Co., Ltd., China) with a $50-\mathrm{kN}$ load. The cross head speed was $5 \mathrm{~mm} / \mathrm{min}$ and the load was applied until the specimens were compressed to about $50 \%$ of their original height or till the samples collapsed. Five replicates were performed for each group and the results are expressed as the means \pm standard deviation $(\mathrm{M} \pm \mathrm{SD})$.

The in vitro degradability of the composites was assessed by soaking the samples $(10 \mathrm{~mm} \times 10 \mathrm{~mm} \times 20 \mathrm{~mm})$ in phosphate buffered saline ( $\mathrm{PBS}, \mathrm{pH}=7.4$ ) for 24 weeks according to the reported methods ${ }^{10}$. After 4, 8, 12, 16 and 24 weeks soaking, the samples were weighed to determine the residual weight and the weight loss was calculated.

The ACP/MAC composite was co-cultured with MG63 cell and the cell morphology, proliferation and differentiation were evaluated according to the reported method ${ }^{8}$.

\section{RESULTS AND DISCUSSION}

FT-IR analysis: The FT-IR spectrum of ACP/MAC is shown in Fig. 1. The peaks at 516 and $1090 \mathrm{~cm}^{-1}$ belong to $\mathrm{PO}_{4}{ }^{3-}$. No peak splitting was observed at $516 \mathrm{~cm}^{-1}$, indicating the as-prepared calcium phosphate was amorphous. The peak of MAC at $3303 \mathrm{~cm}^{-1}$ was attributed to nitrogen- hydrogen(NH) stretching vibrations peak and the bands around 2931 and 2856 $\mathrm{cm}^{-1}$ attributed to carbon hydrogen $\left(\mathrm{CH}_{2}\right)$ vibration peaks. The peaks $1544 \mathrm{~cm}^{-1}$ represented the stretching vibrations of carbon-nitrogen $(\mathrm{CN})$. The band $1646 \mathrm{~cm}^{-1}$ was carbonyl vibration $(\mathrm{C}=\mathrm{O})$. Both the peaks of ACP and those of copolymer were existed in the composite and showed no manifest shift, indicating that the composite was composed of ACP and MAC.

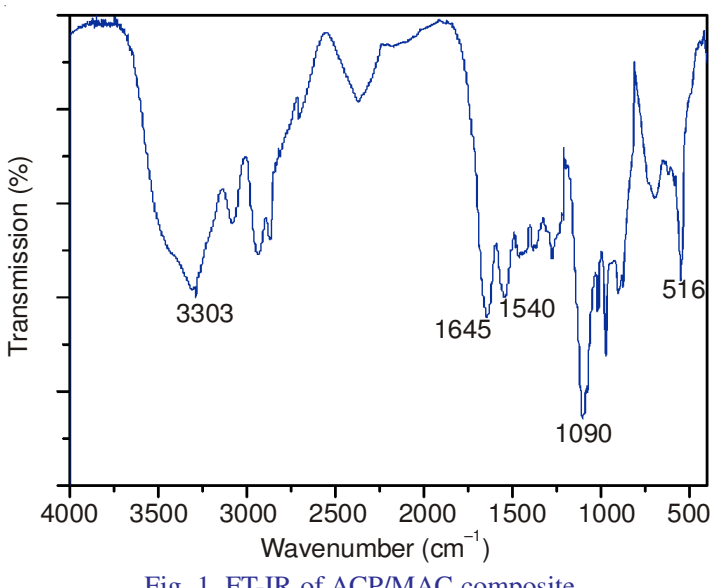

Fig. 1. FT-IR of ACP/MAC composite

Compressive strength: Fig. 2 shows the compressive strength of the ACP/MAC and CDHA/MAC composites. It can be seen that the compressive strength of the ACP/MAC composites increased with an increase in the ACP content and the highest compressive strength of $96 \mathrm{MPa}$ was found for the

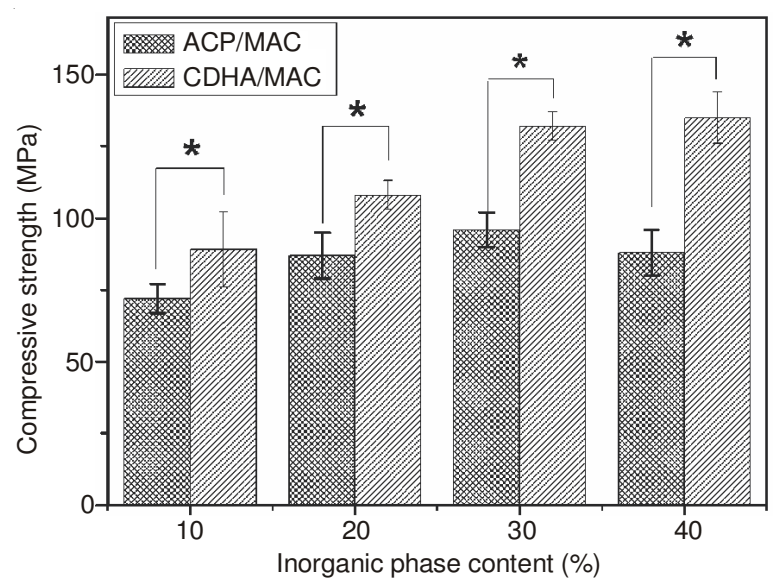

Fig. 2. Compressive strength of ACP/MAC and CDHA/MAC composites

composite with $30 \mathrm{w} \%$ ACP. However, a further increase in the ACP content in the composite to $40 \mathrm{w} \%$ reduced the compressive strength of the composite to $88 \mathrm{MPa}$. All the ACP/ MAC composite samples collapsed during the compressive test, indicating their brittleness. It is obvious that CDHA/MAC shows higher compressive strength of 89-135 MPa compared with ACP/MAC composites, indicating that ACP shows weaker enforcement effect to MAC than CDHA to MAC.

Mechanical test results indicated that the compressive strength of ACP/MAC composite was closed to that of cortical bone of human (50-140 MPa), varied with ACP content in composite $^{12}$. And hence it can meet the fundamental mechanical support requirements. By comparison, the compressive strength of ACP/MAC composite was significantly lower than that of CDHA/MAC composite. This may because ACP filler is weaker and of a lower modulus than glass or ceramic filler ${ }^{13}$.

In vitro degradability: Fig. 3 shows the degradability of ACP/MAC and CDHA/MAC composites in PBS over time. The results show that the weight loss of the ACP/MAC composites increases with soaking time in PBS, indicating that the composites exhibit good degradability in PBS. After soaking for 24 weeks, the weight losses obtained for ACP/MAC with different ACP content were 65, 69, 76 and $84 \mathrm{w} \%$, respectively. These results also show that the ACP content in MAC obviously affected the degradability of the composites. The weight loss of CDHA/MAC composites also increased with increasing soaking time from 0-16 weeks. However, the weight losses of CDHA/MAC composites showed only slightly increased from 17-24 weeks. In contrast, ACP/MAC composites showed higher weight losses than those achieved by CDHA/MAC with the same content of inorganic phase and maintained continuous increase of weight loss during the whole soaking time, suggesting that ACP/MAC could be degraded with high degradation rate and high degradation degree. In our experiments, it was found that all ACP/MAC composite samples were collapsed after 8-week soaking while the CDHA/MAC samples kept integrate shape and only some surface cracks were observed in 40CDHA/MAC samples. Obviously, collapsed samples showed bigger surface area than that of integrate samples, which might be responsible to the high degradation rate and degradation degree of ACP/MAC composite. Bone implants are expected to be degradable at a rate that matches the speed of new tissue formation. Many degradable single composition 

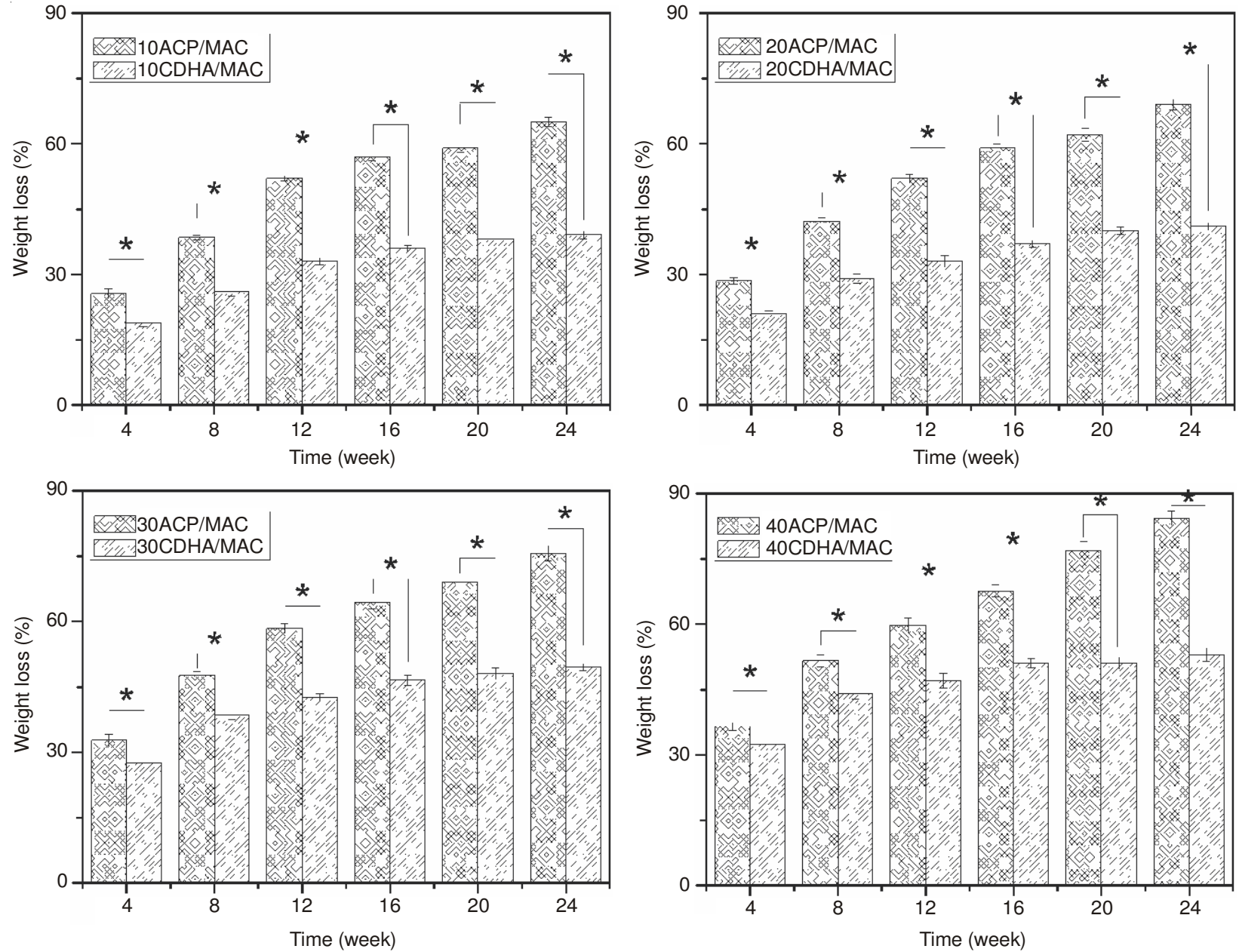

Fig. 3. Weight loss of ACP/MAC composites and CDHA/MAC composites after soaking in PBS

biomaterials have been developed for bone void filler, for examples, calcium sulphate cement, tricalcium phosphate ceramic and hydroxyapatite ceramic ${ }^{4}$. However, those single composition materials show manifest disadvantages. For instance, degradation rate of those materials are not ideal. In addition, they are lack of flexibility and hence are difficult to manipulate in surgery. The ACP/MAC composite showed a relatively stable degradation rate over the soaking period with the weight loss of ACP/MAC composite in the range of 65-84 w \% after 24 weeks soaking in PBS, varying with the ACP content in composite. In addition, ACP/MAC show good initial strength and easy to be processed into desirable shape, indicating that ACP/MAC composite may be better than single composition biomaterials when it is used for bone void filler.

Cytocompatibility: The proliferation of MG63 cells on the 30ACP/MAC composite was evaluated using MTT and 30CDHA/MAC composite was used as a control. As shown in Fig. 4, the OD values of both 30ACP/MAC composite and 30CDHA/MAC composite increased with time, suggesting that MG63 cells were viable on these samples, which exhibited positive cellular responses. The OD values of 30ACP/MAC were higher than those of 30CDHA/MAC at 3,5 and 7 days. However, there was no significant difference in the OD values between 30ACP/MAC composite and 30CDHA/MAC composite.

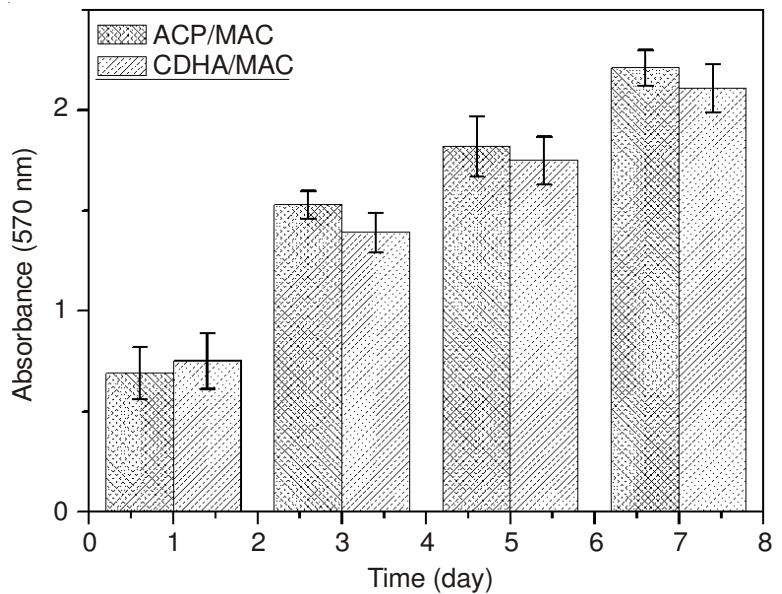

Fig. 4. MG63 cell proliferation on 30ACP/MAC composite and 30CDHA/ MAC composite was used as control

The ALP activity of MG63 cells cultured on the 30ACP/ MAC composite and control were determined and the results are shown in Fig. 5. The ALP activity of the cells on both composites was increased with time. No significant difference in ALP activity was found between the 30ACP/MAC composite and the control after 1,3,5 and 7 days though the level of ALP activity of the cells grown on the ACP/MAC composite was higher than those of the cells grown on CDHA/ MAC composite. 


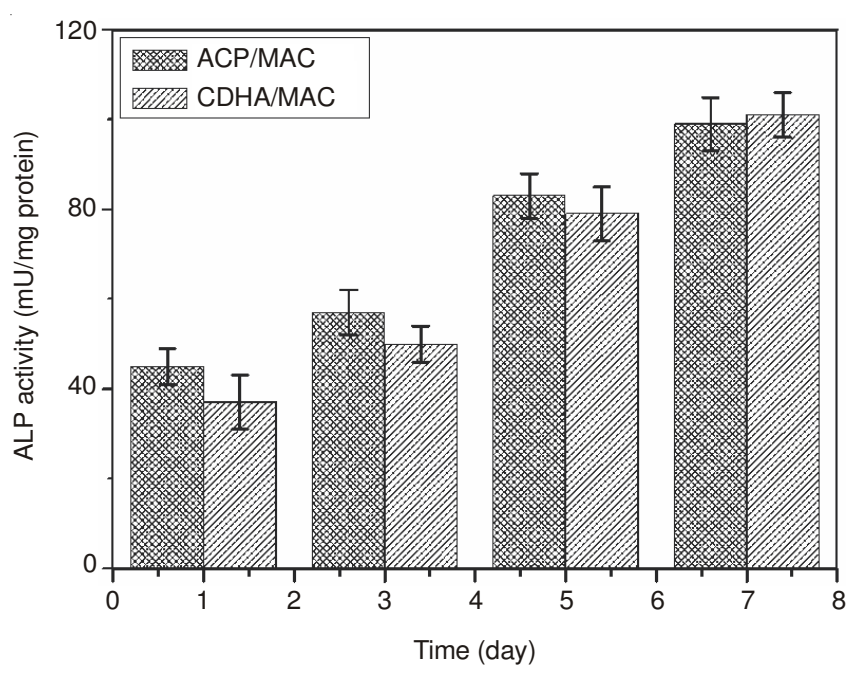

Fig. 5. ALP activity of MG63 cells cultured on 30ACP/MAC composite and 30CDHA/MAC composite was used as a control

Fig. 6 shows the morphology of the MG63 cells cultured on the 30ACP/MAC composite and control for 3 days. The cells were spread on the substrates and linked with each other, which reflects the overall good adherence of the cells to the surfaces of both the ACP/MAC composite and CDHA/MAC composite, i.e., both substrates showed good cytocompatibility. Biocompatibility and safety are the essential requirements for biomaterials to be implanted in vivo. In general, to assess in vitro biocompatibility, ACP/MAC composite was co-cultured with cell and the interactions of cell/material were evaluated. The cell attachment and growth on surface of ACP/MAC composite was observed and the results form SEM images showed that the cells adhered well and reached confluence on the surface of ACP/MAC composite and control after 3 days culture. The MTT assay results showed that the OD values of both ACP/MAC composite and control (CDHA/MAC composite) were increased with co-culture time. The SEM observation and MTT assay results indicated that ACP/MAC composite show little negative effects on MG63 cell growth proliferation and appeared to be biocompatible.

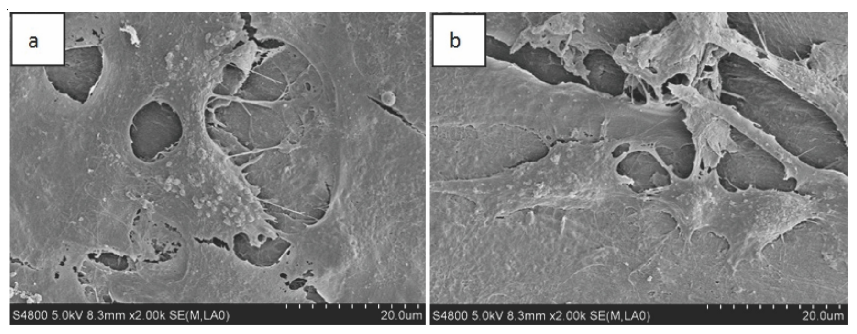

Fig. 6. SEM images of MG63 cells cultured on 30ACP/MAC (a) and 30CDHA/MAC (b) for 3 days
ALP is an enzyme secreted by osteoblasts and hence it is a marker of osteoblastic phenotype and mineralization ${ }^{10}$. The ALP activity of ACP/MAC composite increased with time and was similar to that of the control (CDHA/MAC), which confirmed that ACP/MAC composite supported the osteoblastic differentiation of MG63 cells.

\section{Conclusion}

In this study, a novel ACP/MAC composite was prepared for bone repair. The addition of ACP into MAC could improve the compressive strength of ACP/MAC composite. However, the enforcement effect of ACP to MAC was significantly lower that achieved by CDHA due to its lower modules. In addition, the addition of ACP into MAC changed the degradation rate and kept sustained degradation during the soaking period. The in vitro co-culture of MG63 cells and ACP/MAC composite assessment showed that the composite support the adhesion, proliferation and differentiation of MG63 cells, indicating its good cytocompatibility. Therefore, ACP/MAC composite showed good degradability, biocompatibility and could be easily shaped to fit the defects and may be used for the development of bone void filler for orthopaedic surgery.

\section{ACKNOWLEDGEMENTS}

The authors are grateful for the financial support provided by the Hebei Province Health Department Research Fund mentoring program (No. 20130268).

\section{REFERENCES}

1. T. Uysal, M. Amasyali, S. Ozcan, A.E. Koyuturk, M. Akyol and D. Sagdic, Aust. Dent. J., 55, 285(2010).

2. C. Combes and C. Rey, Acta Biomater, 6, 3362 (2010).

3. G.E. Schumacher, J.M. Antonucci, J.N.R. O'Donnell and D. Skrtic, J. Am. Dent. Assoc., 138, 1476 (2007).

4. R.Z. LeGeros, Chem. Rev., 108, 4742 (2008).

5. G. Balasundaram, M. Sato and T.J. Webster, Biomaterials, 27, 2798 (2006).

6. W.P. Zhang and J.M. Shao, J. Biomed. Mater. Res. A, 94, 450 (2010).

7. W.P. Zhang, Polym. Bull., 60, 323 (2008).

8. H. Li, Y.G. Yan, J. Wei, J. Ma, M. Gong, X.M. Luo and Y.F. Zhang, J. Mater. Sci. Mater. Med., 22, 2555 (2011).

9. Y.F. Zhang, W.P. Shan, X.D. Li, J. Wei, H. Li, J. Ma and Y.G. Yan, Appl. Surf. Sci., 258, 2632 (2012).

10. H. Li, M. Gong, A.P. Yang, J. Ma, X.D. Li and Y.G. Yan, Int. J. Nanomed., 7, 1287 (2012).

11. Z. Ma, F. Chen, Y.J. Zhu, T. Cui and X.Y. Liu, J. Colloid Interf. Sci., 359, 371 (2011).

12. C.C. Verheyen, J.R. de Wijn, C.A. van Blitterswijk and K. de Groot, J. Biomed. Mater. Res., 26, 1277 (1992).

13. J. Wei and Y.B. Li, Eur. Polym. J., 40, 509 (2004). 